\title{
Nonlinear Vibration of a Multirotor System Connected by a Flexible Coupling Subjected to the Holonomic Constraint of Dynamic Angular Misalignment
}

\begin{abstract}
M. Li
Department of Mechanics, Xi'an University of Science and Technology, Xi'an 710054, China

Correspondence should be addressed to M. Li, limingnuaa@hotmail.com

Received 1 September 2011; Revised 28 November 2011; Accepted 13 December 2011

Academic Editor: Alex Elias-Zuniga

Copyright (C) 2012 M. Li. This is an open access article distributed under the Creative Commons Attribution License, which permits unrestricted use, distribution, and reproduction in any medium, provided the original work is properly cited.

This paper proposes a mathematical model of the multirotor system with a flexible coupling on spring supports on Lagrange's approach, which has taken into account the effects of dynamic angular misalignment and mass unbalance. Then its nonlinear dynamic behaviors of the system are discussed based on the method of multiple scales and numerical technique, respectively. The results show that the responses of the system in lateral directions contain a similar component to that of the mass unbalanced system on both the vibrating frequency and amplitude and involve the typical nonlinear components such as the ones from some combined frequencies; the results also reveal that the numerical agreements on the above-mentioned methods are perfect for the transient responses.
\end{abstract}

\section{Introduction}

The misalignment of rotor system is one of the most common mechanical faults in rotating machinery, which can cause excessive vibrations, produce bothering noises, and create damaging forces on rotors, bearings, and couplings. For large-scale rotating system, there are several reasons that lead to the misalignments such as the various deformations of rotors, uncoaxiality of bearings and couplings. According to the geometric relationship between the two rotors, the types of misalignment may be divided into the parallel and angle ones or their combination. In practical engineering, generally the misalignment appears in some main form, for example, the ground subsidence or looseness and improper assembly of the rotor system can induce the faults of angle misalignment. In fact, even a multirotor system is well aligned in static equilibrium state; it still presents the dynamic misalignment or offset in a rotor system which couples by a flexible coupling. 
During the past decades, the dynamics of effects on the rotor misalignments has been studied in a variety of aspects, that is, firstly Gibbons [1] established a model to predict the additional forces due to the misalignment of the gear coupling, Dewell and Mitchell [2] analyzed the lateral vibration frequencies for a misaligned rotor system which were based on the experiment, and $\mathrm{Li}$ and $\mathrm{Yu}$ [3] discussed the coupled lateral and torsional vibration of a rotor-bearing system with the misalignment by a gear coupling. In paper [4], Al-Hussain and Redmond studied the effect of parallel misalignment on the lateral and torsional responses of two-rotor system, and in [5], Al-Hussain considered the effect of angular misalignment on the stability of rotors connected by a flexible mechanical coupling. Prabhakar et al. [6] dealt with the transient responses of a rotor system with a rotor misalignment by using the finite element method. Recently, Hili et al. [7] established a linear dynamic model of a multirotor system which couples a Cardan joint by using the finite element method, in which both of the faults misalignment and unbalance were considered. Lees [8] studied the misalignment of a rigidly coupled rotor which mounted on idealized linear bearings with a linear time-varying stiffness. Li [9] developed a mathematical model of a multirotor system by taking into account the effects of parallel misalignment and unbalance of rotors, in which the misalignment was dealt with as a holonomic constraint, and Slim et al. [10] paid attention to the dynamic behaviors of misaligned rotor system mounted on two journal bearings. Patel and Darpe [11] investigated vibrating responses of misaligned rotors modeled by using Timoshenko beam elements with six degrees of freedom, and in paper [12], they studied the frequency spectra of the system supported on rolling element bearings by experiment. In 2010, Redmond [13] developed a dynamic modal of misaligned shafts, which included both the static angular and parallel misalignment.

For the multirotor system, flexible couplings are commonly used to transmit torque from one rotor to another when the adjacent shafts are slightly misaligned. And yet it is required coaxially during the installation for avoiding the additional forces on bearings, for example, the force is not permitted to be produced for a flexible coupling under static conditions; however, it is aligned or coaxial in this state, the coupling still appears a dynamic misalignment at work [14], that is, a dynamic angular deformation is generated on the flexible coupling duo to the dynamic load. In the above discussions, while much work has been done on the dynamics of the system that an initial or static misalignment exists, the little attention has been paid to the dynamic one. Accordingly, the purpose of the present paper is to explain the vibration mechanism and dynamic characteristics of rotor system with a flexible coupling after considering the effect on the dynamic angular misalignment.

\section{Motion Equations}

The proposed model of the multirotor system connected by a flexible coupling is shown in Figure 1, in which $m_{1}$ and $m_{2}$ denote the lumped masses, respectively; $k_{1}$ and $k_{2}$ represent the stiffness of the bearings; $k_{t}$ is the equivalent angular stiffness for the flexible couple. For simplification, the following assumptions upon the system of concern will be used hereinafter: (1) the two rotors are rigid; (2) the bearings are isotropic on dynamic performances; (3) the misaligned angle between the adjacent rotors is small.

The coordinate system oxyz is set up in the static equilibrium position of rotor system as shown in Figure 1. Let $O_{1}\left(x_{1}, y_{1}, 0\right)$ and $O_{2}\left(x_{2}, y_{2}, z_{2}\right)$ be the coordinates of the mass 


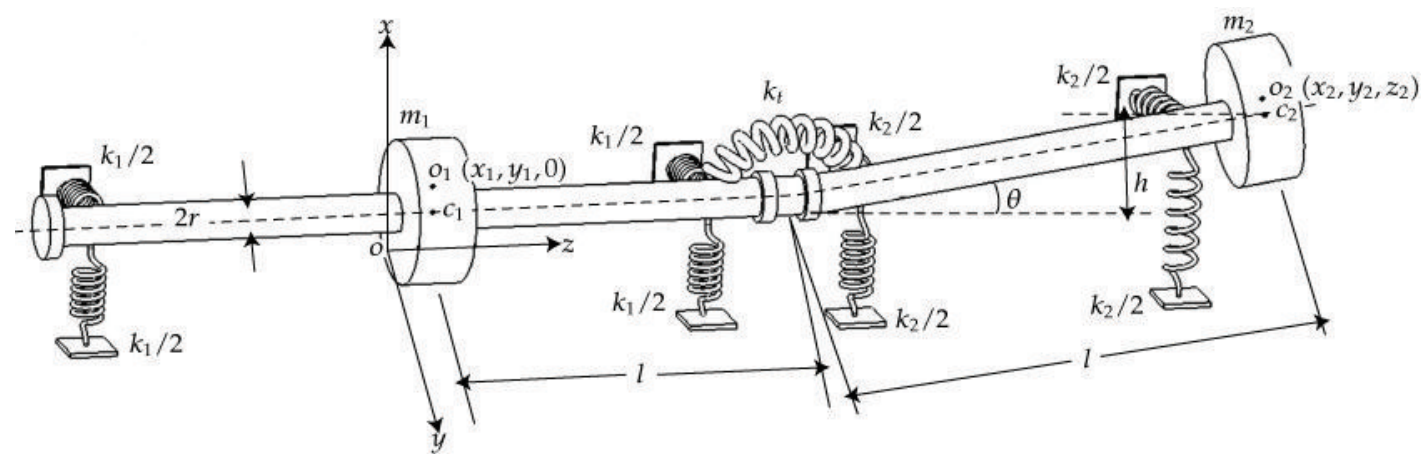

Figure 1: Schematic diagram of angular-misaligned rotor system.

centers of disc 1 and disc 2, respectively, then they yield

$$
\begin{gathered}
x_{1}=x+e_{1} \cos (\Omega t), \\
y_{1}=y+e_{1} \sin (\Omega t), \\
z_{1}=0, \\
x_{2}=x+l \sin \theta \cos (\Omega t)+e_{2} \cos \theta \cos (\Omega t+\gamma), \\
y_{2}=y+l \sin \theta \sin (\Omega t)+e_{2} \cos \theta \sin (\Omega t+\gamma), \\
z_{2}=l+l \cos \theta,
\end{gathered}
$$

where $x$ and $y$ are the displacements of disc 1 at the geometric center $C_{1} ; \theta$ is the angle between adjacent rotors; $\Omega$ is the rotating speed; $\gamma$ is the initial phase angle; $l$ is the rotor length; $e_{1}, e_{2}$ are the mass unbalances. If the generalized coordinates $x, y$, and $\theta$ are introduced, then (2.2) describes a nonstationary holonomic constraint because the displacement varies with time $t$. Generally, the offset $h$ of disc 2 is larger than $e_{2}$; therefore, the terms related to $e_{2}$ are ignored in the paper.

The kinetic energy of the system can be written as

$$
T=\sum_{i=1}^{2} \frac{1}{2} m_{i}\left(\dot{x}_{i}^{2}+\dot{y}_{i}^{2}+\dot{z}_{i}^{2}\right)
$$

and the potential energy is

$$
U=\sum_{i=1}^{2} \frac{1}{2} k_{i}\left(x_{i}^{2}+y_{i}^{2}\right)+\frac{1}{2} k_{t} \theta^{2} .
$$

For simplifying the representation furthermore, let $m_{1}=m_{2}=m, k_{1}=k_{2}=k$, and $e_{1}=e$, then substituting (2.1) and (2.2) into (2.3) and (2.4), and based on Lagrange's equation

$$
\frac{d}{d t}\left(\frac{\partial T}{\partial \dot{q}_{j}}\right)-\frac{\partial T}{\partial q_{j}}+\frac{\partial U}{\partial q_{j}}=0, \quad q_{j}=\left[\begin{array}{lll}
x & y & \theta
\end{array}\right]^{T},
$$


the motion equation in the generalized coordinates becomes

$$
\begin{aligned}
& 2 m \ddot{x}+m l \ddot{\theta} \cos \theta \cos \Omega t-m l \dot{\theta}^{2} \sin \theta \cos \Omega t-2 m l \Omega \dot{\theta} \cos \theta \sin \Omega t-m l \Omega^{2} \sin \theta \cos \Omega t \\
&+2 k x+k l \sin \theta \cos \Omega t=m e \Omega^{2} \cos \Omega t, \\
& 2 m \ddot{y}+m l \ddot{\theta} \cos \theta \sin \Omega t-m l \dot{\theta}^{2} \sin \theta \sin \Omega t+2 m l \Omega \dot{\theta} \cos \theta \cos \Omega t-m l \Omega^{2} \sin \theta \sin \Omega t \\
& \quad+2 k y+k l \sin \theta \sin \Omega t=m e \Omega^{2} \sin \Omega t, \\
& m l^{2} \ddot{\theta}+m l \ddot{x} \cos \theta \cos \Omega t+m l \ddot{y} \cos \theta \sin \Omega t-m l^{2} \Omega^{2} \sin \theta \cos \theta+k l x \cos \theta \cos \Omega t \\
& \quad+k l y \cos \theta \sin \Omega t+k l^{2} \sin \theta \cos \theta+k_{t} \theta=0 .
\end{aligned}
$$

The above equations are second-order ordinary differential ones with variable coefficients, which is obviously the characteristic of strong nonlinearity. Based on the theory of differential equations, it is difficult to solve. By applying the inverse operation, (2.6) can be expressed as

$$
\begin{aligned}
& \ddot{x}-\frac{1}{m l\left(1+\sin ^{2} \theta\right)}\left[m l^{2} \dot{\theta}^{2} \sin \theta \cos \Omega t+2 m l^{2} \Omega \dot{\theta} \cos \theta \sin \Omega t-m l^{2} \Omega \dot{\theta} \cos ^{3} \theta \sin \Omega t\right. \\
& +m l^{2} \Omega^{2} \sin ^{3} \theta \cos \Omega t-k l^{2} \sin ^{3} \theta \cos \Omega t-k l x\left(1+\sin ^{2} \theta\right)+k_{t} \theta \cos \theta \cos \Omega t \\
& \left.+m e \Omega^{2} l \cos \Omega t\right]=0, \\
& \ddot{y}-\frac{1}{m l\left(1+\sin ^{2} \theta\right)}\left[m l^{2} \dot{\theta}^{2} \sin \theta \sin \Omega t-2 m l^{2} \Omega \dot{\theta} \cos \theta \cos \Omega t+m l^{2} \Omega \dot{\theta} \cos ^{3} \theta \cos \Omega t\right. \\
& +m l^{2} \Omega^{2} \sin ^{3} \theta \sin \Omega t-k l^{2} \sin ^{3} \theta \sin \Omega t-k l y\left(1+\sin ^{2} \theta\right)+k_{t} \theta \cos \theta \sin \Omega t \\
& \left.+m e \Omega^{2} l \sin \Omega t\right]=0 \\
& \ddot{\theta}+\frac{1}{m l^{2}\left(1+\sin ^{2} \theta\right)}\left[m l^{2} \dot{\theta}^{2} \sin \theta \cos \theta-m l^{2} \Omega^{2} \sin \theta \cos \theta+k l^{2} \sin \theta \cos \theta+2 k_{t} \theta\right. \\
& \left.+m e \Omega^{2} l \cos \theta\right]=0
\end{aligned}
$$

Let $X=x / r, Y=y / r$ be the nondimensional displacements, in which $r$ is the radius of rotor; $E=e / r, L=l / r$ are the nondimensional mass eccentricity and rotor length, respectively; $\tau=\Omega t$ is the nondimensional time; $\omega_{0}=\sqrt{k / m \Omega^{2}}, \omega_{t}=\sqrt{k_{t} / m l^{2} \Omega^{2}}$ are the nondimensional angular frequencies and denote $d x / d t=x^{\prime}(d X / d \tau)=X^{\prime} \ldots$; accordingly, (2.7) can be cast into the following nondimensional form:

$$
\begin{gathered}
X^{\prime \prime}-\frac{1}{\left(1+\sin ^{2} \theta\right)}\left[L\left(\theta^{\prime}\right)^{2} \sin \theta \cos \tau+2 L \theta^{\prime} \cos \theta \sin \tau-L \theta^{\prime} \cos ^{3} \theta \sin \tau-\left(\omega_{0}^{2}-1\right) L \sin ^{3} \theta \cos \tau\right. \\
\left.-\omega_{0}^{2} X\left(1+\sin ^{2} \theta\right)+\omega_{t}^{2} L \theta \cos \theta \cos \tau+E \cos \tau\right]=0
\end{gathered}
$$




$$
\begin{aligned}
Y^{\prime \prime}-\frac{1}{\left(1+\sin ^{2} \theta\right)}\left[L\left(\theta^{\prime}\right)^{2} \sin \theta \sin \tau-2 L \theta^{\prime} \cos \theta \cos \tau+L \theta^{\prime} \cos ^{3} \theta \cos \tau\right. \\
\left.-\left(\omega_{0}^{2}-1\right) L \sin ^{3} \theta \sin \tau-\omega_{0}^{2} Y\left(1+\sin ^{2} \theta\right)+\omega_{t}^{2} L \theta \cos \theta \sin \tau+E \sin \tau\right]=0, \\
\theta^{\prime \prime}+\frac{1}{\left(1+\sin ^{2} \theta\right)}\left[\left(\theta^{\prime}\right)^{2} \sin \theta \cos \theta+\left(\omega_{0}^{2}-1\right) \sin \theta \cos \theta+2 \omega_{t}^{2} \theta+\frac{E}{L} \cos \theta\right]=0 .
\end{aligned}
$$

Equation (2.8) is a parametrically excited system with three degrees of freedom on the theory of nonlinear vibration, in which $\omega_{0}$ and $\omega_{t}$ are the frequency ratios, respectively; $\varepsilon$ is the nondimensional mass eccentricity $L$ is the nondimensional length. In the above equation, the third one is decoupled with the first two equations, that is, the generalized coordinate $\theta$ is totally independent of $X$ and $Y$ thereby it can be solved in the first place.

In the case of the initial angular misalignment, it can be assumed that $\theta=\alpha+\varphi(t)$ in which $\alpha$ is the constant angle and $\varphi(t)$ is its perturbation. After substituting $\theta$ into (2.8) and expanding the relevant terms in the Taylor series, after neglecting the higher-order ones the governing equations that is of the initial or static angular misalignment between two rotors are obtained

$$
\begin{gathered}
X^{\prime \prime}-\frac{1}{\left(1+\sin ^{2} \alpha\right)}\left[L\left(\varphi^{\prime}\right)^{2} \sin \alpha \cos \tau+L \varphi^{\prime}\left(1+\sin ^{2} \alpha\right) \cos \alpha \sin \tau-\left(\omega_{0}^{2}-1\right) L \sin ^{3} \alpha \cos \tau\right. \\
\left.-\omega_{0}^{2}\left(1+\sin ^{2} \alpha\right) X+\omega_{t}^{2} L \alpha \cos \alpha \cos \tau+E \cos \tau\right] \\
-\frac{\varphi}{\left(1+\sin ^{2} \alpha\right)}\left[L\left(\varphi^{\prime}\right)^{2} \cos \alpha \cos \tau+L \varphi^{\prime}\left(3 \cos ^{2} \alpha-2\right) \sin \alpha \sin \tau-2 \omega_{0}^{2} X \cos \alpha \sin \alpha\right. \\
\left.-\omega_{t}^{2} L(\alpha \sin \alpha-\cos \alpha) \cos \tau+3 L\left(1-\omega_{0}^{2}\right) \sin ^{2} \alpha \cos \alpha \cos \tau\right] \\
+\frac{\varphi \sin 2 \alpha}{\left(1+\sin ^{2} \alpha\right)^{2}}\left[L\left(\varphi^{\prime}\right)^{2} \sin \alpha \cos \tau+L \varphi^{\prime}\left(1+\sin ^{2} \alpha\right) \cos \alpha \sin \tau+\omega_{t}^{2} \alpha L \cos \alpha \cos \tau\right. \\
\left.-\omega_{0}^{2}\left(1+\sin ^{2} \alpha\right) X+L\left(1-\omega_{0}^{2}\right) \sin ^{3} \alpha \cos \tau+E \cos \tau\right]=0, \\
Y^{\prime \prime}-\frac{1}{\left(1+\sin ^{2} \alpha\right)}\left[L\left(\varphi^{\prime}\right)^{2} \sin \alpha \sin \tau-L \varphi^{\prime}\left(1+\sin ^{2} \alpha\right) \cos \alpha \cos \tau-\left(\omega_{0}^{2}-1\right) L \sin { }^{3} \alpha \sin \tau\right. \\
\left.-\omega_{0}^{2}\left(1+\sin ^{2} \alpha\right) Y+\omega_{t}^{2} L \alpha \cos \alpha \sin ^{2}+E \sin \tau\right] \\
-\frac{\varphi}{\left(1+\sin ^{2} \alpha\right)}\left[L\left(\varphi^{\prime}\right)^{2} \cos \alpha \sin \tau+L \varphi^{\prime}\left(2-3 \cos ^{2} \alpha\right) \sin \alpha \cos \tau-2 \omega_{0}^{2} Y \cos \alpha \sin \alpha\right. \\
\left.-\omega_{t}^{2} L(\alpha \sin \alpha-\cos \alpha) \sin \tau+3 L\left(1-\omega_{0}^{2}\right) \sin { }^{2} \alpha \cos \alpha \sin \tau\right] \\
-\frac{\varphi \sin ^{2} \alpha}{\left(1+\sin ^{2} \alpha\right)^{2}}\left[L\left(\varphi^{\prime}\right)^{2} \sin \alpha \sin \tau-L \varphi^{\prime}\left(1+\sin ^{2} \alpha\right) \cos \alpha \cos \tau+\omega_{t}^{2} \alpha L \cos \alpha \sin \tau\right. \\
\left.\left.-\sin ^{2} \alpha\right) Y+L\left(1-\omega_{0}^{2}\right) \sin ^{3} \alpha \sin \tau+E \sin \tau\right]=0,
\end{gathered}
$$




$$
\begin{gathered}
\varphi^{\prime \prime}+\frac{1}{\left(1+\sin ^{2} \alpha\right)}\left[\left(\varphi^{\prime}\right)^{2} \sin \alpha \cos \alpha+2 \omega_{t}^{2} \alpha+\left(\omega_{0}^{2}-1\right) \sin \alpha \cos \alpha+\frac{E}{L} \cos \alpha\right] \\
+\frac{\varphi}{\left(1+\sin ^{2} \alpha\right)}\left\{\left(\varphi^{\prime}\right)^{2} \cos 2 \alpha+2 \omega_{t}^{2}+\left(\omega_{0}^{2}-1\right) \cos 2 \alpha-\frac{E}{L} \sin \alpha\right. \\
-\frac{\varphi \sin 2 \alpha}{\left(1+\sin ^{2} \alpha\right)^{2}}\left[\left(\varphi^{\prime}\right)^{2} \sin \alpha \cos \alpha+2 \omega_{t}^{2} \alpha+\left(\omega_{0}^{2}-1\right) \sin \alpha \cos \alpha\right. \\
\left.\left.+\frac{E}{L} \cos \alpha\right]\right\}=0 .
\end{gathered}
$$

\section{The Approximation Solution}

For discussing the vibration mechanism and characteristics of the system conveniently, let $\alpha=$ 0 , namely, the initial or static angular misalignment of a flexible coupling vanishes, and only the dynamic angular misalignment presents in the system, then (2.9) reduces to

$$
\begin{gathered}
X^{\prime \prime}-L\left(\varphi^{\prime}\right)^{2} \varphi \cos \tau-L \varphi^{\prime} \sin \tau+\omega_{0}^{2} X-\omega_{t}^{2} L \varphi \cos \tau=E \cos \tau, \\
Y^{\prime \prime}-L\left(\varphi^{\prime}\right)^{2} \varphi \sin \tau+L \varphi^{\prime} \cos \tau+\omega_{0}^{2} Y-\omega_{t}^{2} L \varphi \sin \tau=E \sin \tau, \\
\varphi^{\prime \prime}+\left(\varphi^{\prime}\right)^{2} \varphi+\left(\omega_{0}^{2}+2 \omega_{t}^{2}-1\right) \varphi+\frac{E}{L}=0 .
\end{gathered}
$$

The third of (3.1) is uncoupled with the first two; therefore, it can be simplified as

$$
\phi^{\prime \prime}+\left(\phi^{\prime}\right)^{2} \phi+\varpi_{0}^{2} \phi=0
$$

where $\phi=\varphi+E / L \varpi^{2}, \varpi_{0}^{2}=\omega_{0}^{2}+2 \omega_{t}^{2}-1$.

The method of multiple scales (MMSs) is introduced for the approximation solution of the nonlinear vibration in this work. For the small angle $\phi$, let $T=\omega \tau$ and expand $\phi$ and $\omega$ $[15]$ as

$$
\begin{gathered}
\phi(T, \varepsilon)=\varepsilon \phi_{1}(T)+\varepsilon^{3} \phi_{3}(T), \\
\omega=\varpi_{0}+\varepsilon^{2} \omega_{2},
\end{gathered}
$$

where $\varepsilon$ stands for a small nondimensional parameter characterizing the amplitude of the motion. The term $\varepsilon^{2} \phi_{2}(\tau)$ is missing from (3.3) because the nonlinearity appears at $O\left(\varepsilon^{3}\right)$. The term $\varepsilon \omega_{1}$ is absent from (3.4) because the frequency is independent of the sign of $\varepsilon$. Substituting (3.3) and (3.4) into (3.2) and equating coefficients of like powers of $\varepsilon$, after donating $d \phi / d T=\phi^{*}$, it yields

$$
\begin{gathered}
\varpi_{0}^{2}\left(\phi_{1}^{* *}+\phi_{1}\right)=0 \\
\varpi_{0}^{2}\left(\phi_{3}^{* *}+\phi_{3}\right)=-2 \varpi_{0} \omega_{2} \phi_{1}^{* *}-\varpi_{0}^{2}\left(\phi_{1}^{*}\right)^{2} \phi_{1} .
\end{gathered}
$$


The solution of (3.5) is of the form

$$
\phi_{1}=a \cos (T+\beta),
$$

where $a$ and $\beta$ are constants; hence, (3.6) becomes

$$
\phi_{3}^{* *}+\phi_{3}=\left(\frac{\omega_{2}}{\varpi_{0}}-\frac{1}{4} a^{2}\right) a \cos (T+\beta)+\frac{1}{4} a^{3} \cos (3 T+3 \beta)
$$

Eliminating the secular term in (3.8) gives $\omega_{2}=(1 / 4) \varpi_{0} a^{2}$, then disregarding the solution of the homogeneous equation, its solution reduces to

$$
\phi_{3}=-\frac{a^{3}}{32} \cos (3 T+3 \beta)
$$

From the equation $\phi=\varphi+E / L \varpi^{2}$, it follows that

$$
\varphi=-\frac{E}{L \varpi_{0}^{2}}+\varepsilon a \cos \psi-\frac{\varepsilon^{3} a^{3}}{32} \cos 3 \psi,
$$

in which $\psi=\omega \tau+\beta, \omega=\varpi_{0}\left(1+\varepsilon^{2} a^{2} / 4\right)$. If imposing the initial conditions $\left.\varphi\right|_{\tau=0}=0$ and $\left.\varphi^{*}\right|_{\tau=0}=0$, then the constants $\varepsilon a$ and $\beta$ are obtained

$$
\begin{gathered}
\varepsilon a \cos \beta-\frac{\varepsilon^{3} a^{3}}{32} \cos 3 \beta=\frac{E}{L \varpi_{0}^{2}}, \\
\sin \beta\left(1+\frac{3}{32} \varepsilon^{2} a^{2}-\frac{3}{8} \varepsilon^{2} a^{2} \cos ^{2} \beta\right)=0 .
\end{gathered}
$$

When $\beta=0, \varepsilon a$ yields

$$
\varepsilon a-\frac{\varepsilon^{3} a^{3}}{32}=\frac{E}{L \varpi_{0}^{2}}
$$

In practice, $E / L \varpi_{0}^{2}$ is small, thus the solution becomes $\beta=0, \varepsilon a \approx E / L \varpi_{0}^{2}$. For example, if the parameters $L=20.0$ and $E=0.03,0.05$, the errors between them can be neglected at all. The numerical results are shown in Figure 2, which indicate a good agreements under the concerned parameters.

Generally for the above nonlinear equations, there are six real solutions, in which the $\operatorname{root} \beta=0, \varepsilon a \approx E / L \varpi^{2}$ is only satisfied for small oscillation in engineering, that is, the systematic parameters are considered as $\omega_{0}=1.2, \omega_{t}=0.02, L=20.0$, and $E=0.05$, accordingly $\varpi_{0}=\sqrt{\omega_{0}^{2}+2 \omega_{t}^{2}-1}=0.664$, and after solving (3.11) on Maple, the six real roots of 


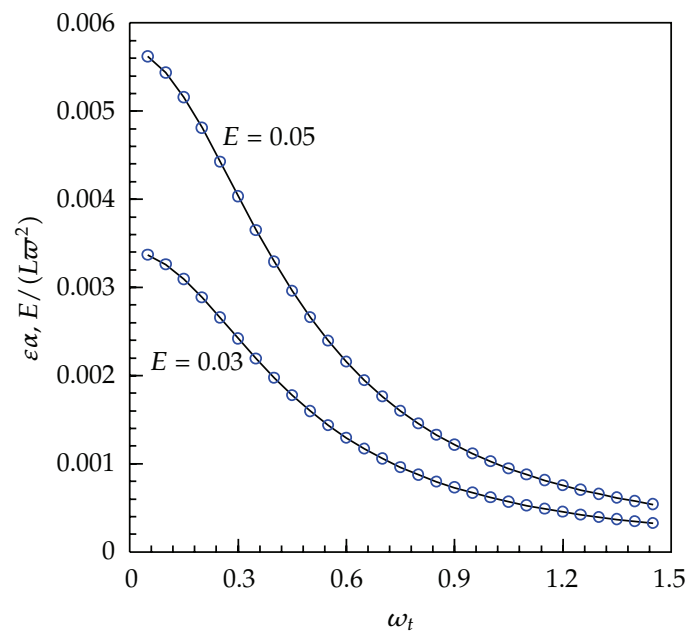

- $\varepsilon \alpha$

$-E / L \varpi^{2}$

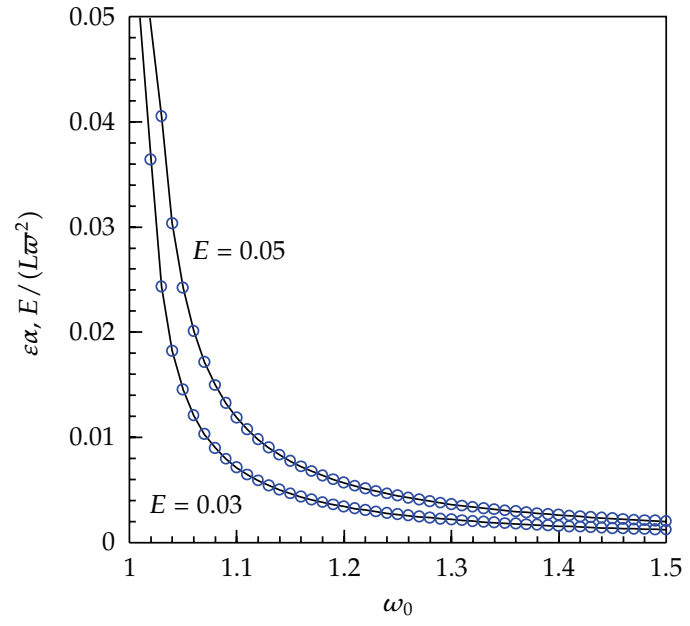

- $\varepsilon \alpha$

$-E / L \varpi^{2}$
(a) $\omega_{0}=1.2$
(b) $\omega_{t}=0.02$

Figure 2: Numerical results for $\varepsilon a$ and $E / L \varpi^{2}$ with increasing $\omega_{0}$ or $\omega_{t}$.

(3.12) are

$$
\begin{array}{r}
\beta=0, \quad \varepsilon a=0.00567 \approx \frac{E}{L \varpi^{2}}, \\
\beta=0, \quad \varepsilon a=-5.65969, \\
\beta=0, \quad \varepsilon a=5.65402, \\
\beta=\pi, \quad \varepsilon a=-0.00567 \approx-\frac{E}{L \varpi^{2}}, \\
\beta=\pi, \quad \varepsilon a=5.65969, \\
\beta=\pi, \quad \varepsilon a=-5.65402 .
\end{array}
$$

The proves that $\beta=0, \varepsilon a \approx E / L \varpi^{2}$ is an available approximate solution, $\beta=0$, and $\varepsilon a=$ $-5.696,5.65402$ are not almost changed with increasing the parameters $\omega_{0}$ and $\omega_{t}$, which are not suitable for the engineering. Thereby, the former will be paid more attention to the following analysis, then expression (3.10) leads to

$$
\varphi=\frac{E}{L \varpi_{0}^{2}}(\cos (\omega \tau)-1)-\frac{1}{32}\left(\frac{E}{L \varpi_{0}^{2}}\right)^{3} \cos (3 \omega \tau)
$$




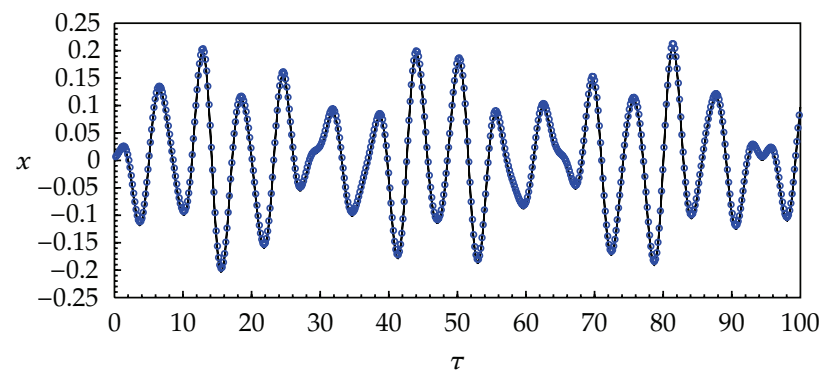

(a)

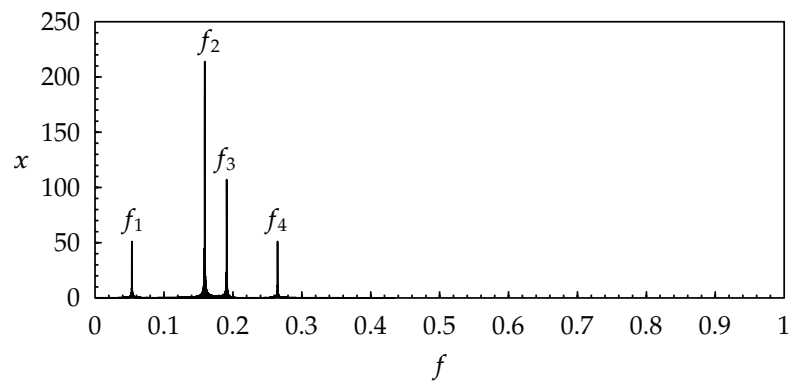

- Approximate solution

— Numerical solution

(b)

Figure 3: The nondimensional response and its frequency spectrum in $x$ direction when $E=0.05$ : (a) response; (b) spectrum.

Substituting expression (3.14) into (3.1), it yields

$$
\begin{aligned}
& X^{\prime \prime}+\omega_{0}^{2} X=E \frac{\omega_{0}^{2}+\omega_{t}^{2}-1}{\varpi_{0}^{2}} \cos (\tau)+\frac{E}{\varpi_{0}^{2}}\left(\omega_{t}^{2} \cos (\omega \tau) \cos (\tau)-\omega \sin (\omega \tau) \sin (\tau)\right)+O\left(E^{2}\right) \\
& Y^{\prime \prime}+\omega_{0}^{2} Y=E \frac{\omega_{0}^{2}+\omega_{t}^{2}-1}{\varpi_{0}^{2}} \sin \tau+\frac{E}{\varpi_{0}^{2}}\left(\omega_{t}^{2} \cos (\omega \tau) \sin (\tau)+\omega \sin (\omega \tau) \cos (\tau)\right)+O\left(E^{2}\right)
\end{aligned}
$$

and its general solution gives

$$
\begin{aligned}
X= & C_{1} \cos \omega_{0} \tau+C_{2} \sin \omega_{0} \tau+E \frac{\omega_{0}^{2}+\omega_{t}^{2}-1}{\varpi_{0}^{2}\left(\omega_{0}^{2}-1\right)} \cos \tau \\
& +\frac{E}{2 \varpi_{0}^{2}}\left(\frac{\omega_{t}^{2}+\omega}{\omega_{0}^{2}-(\omega+1)^{2}} \cos (\omega+1) \tau+\frac{\omega_{t}^{2}-\omega}{\omega_{0}^{2}-(\omega-1)^{2}} \cos (\omega-1) \tau\right)+O\left(E^{2}\right)
\end{aligned}
$$




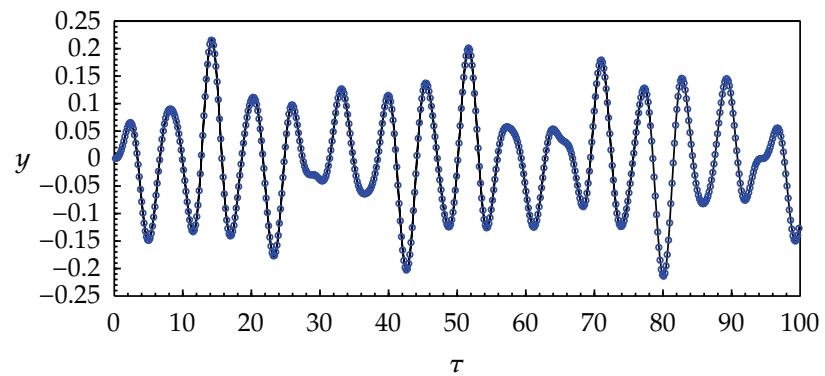

(a)

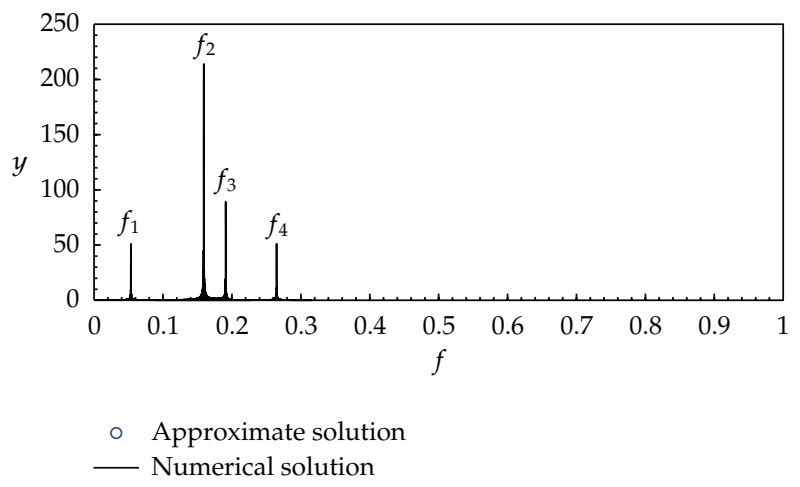

(b)

Figure 4: The nondimensional response and its frequency spectrum in $y$ direction when $E=0.05$ : (a) response; (b) spectrum.

$$
\begin{aligned}
Y= & C_{3} \cos \omega_{0} \tau+C_{4} \sin \omega_{0} \tau+E \frac{\omega_{0}^{2}+\omega_{t}^{2}-1}{\varpi_{0}^{2}\left(\omega_{0}^{2}-1\right)} \sin \tau \\
& +\frac{E}{2 \varpi_{0}^{2}}\left(\frac{\omega_{t}^{2}+\omega}{\omega_{0}^{2}-(\omega+1)^{2}} \sin (\omega+1) \tau-\frac{\omega_{t}^{2}-\omega}{\omega_{0}^{2}-(\omega-1)^{2}} \sin (\omega-1) \tau\right)+O\left(E^{2}\right),
\end{aligned}
$$

where $\omega=\sqrt{\omega_{0}^{2}+2 \omega_{t}^{2}-1}\left(1+\left(E / 2 L \varpi^{2}\right)^{2}\right)$, and $C_{1}, C_{2}, C_{3}$, and $C_{4}$ are the constants.

If the initial conditions $X(0)=X^{\prime}(0)=0$ and $Y(0)=Y^{\prime}(0)=0$ are introduced, the constants $C_{1}, C_{2}, C_{3}$, and $C_{4}$ can be determined easily; therefore, the responses can be shown to reduce to

$$
\begin{aligned}
& X=-(A+B+D) \cos \omega_{0} \tau+A \cos \tau+B \cos (\omega+1) \tau+D \cos (\omega-1) \tau \\
& Y=\frac{D(\omega-1)-B(\omega+1)-A}{\omega_{0}} \sin \omega_{0} \tau+A \sin \tau+B \sin (\omega+1) \tau-D \sin (\omega-1) \tau
\end{aligned}
$$

in which $A=E\left(\left(\omega_{0}^{2}+\omega_{t}^{2}-1\right) / \varpi_{0}^{2}\left(\omega_{0}^{2}-1\right)\right), \quad B=E\left(\omega_{t}^{2}+\omega\right) / 2 \varpi_{0}^{2}\left(\omega_{0}^{2}-(\omega+1)^{2}\right)$, and $D=$ $E\left(\omega_{t}^{2}-\omega\right) / 2 \varpi_{0}^{2}\left(\omega_{0}^{2}-(\omega-1)^{2}\right)$. 


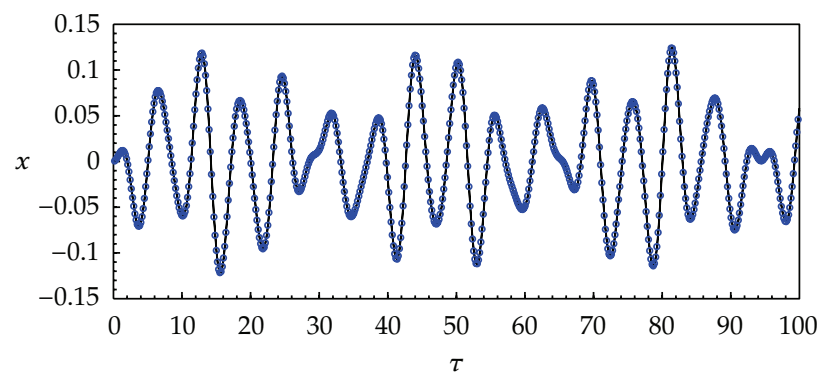

(a)

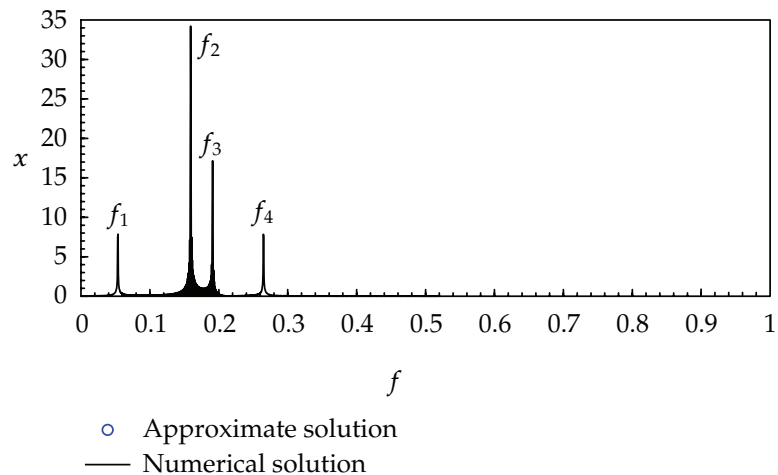

(b)

Figure 5: The nondimensional response and its frequency spectrum in $x$ direction when $E=0.03$ : (a) response; (b) spectrum.

The above solution shows that the response consists of the following components: (a) the free vibration at natural frequency $\omega_{0}$ of the derived linear system. If the damping is concerned in $x$ and $y$ direction, its amplitude will be decreased exponentially on the theory of vibration; (b) forced vibration excited by the mass unbalance at the frequency of rotating speed. When $\omega_{0}=1$, that is, the rotating speed coincides with the lateral natural frequency, the bending resonance of rotor system occurs; (c) the angular motions due to the flexible coupling, in which the frequencies $\omega-1$ and $\omega+1$ are combined by $\omega$ and the rotating speed. It is a typical nonlinear oscillation because the combination resonances exist at $\omega_{0}=$ $\omega-1$ and $\omega_{0}=\omega+1$.

Certainly, it should be noted that the above solution only reveals some dynamic behaviors of the rotor system connected to a flexible coupling, and some other characteristics such as the subharmonic resonances may be emerged if suitable conditions are satisfied.

\section{Numerical Analysis}

Because (2.8) is a strongly nonlinear system, the numerical technique is preferable. Accordingly, the Runge-Kutta method is carried out to predict the dynamic characteristics in the present paper. As a nonautonomous system, traditionally (2.8) is discussed on the state 


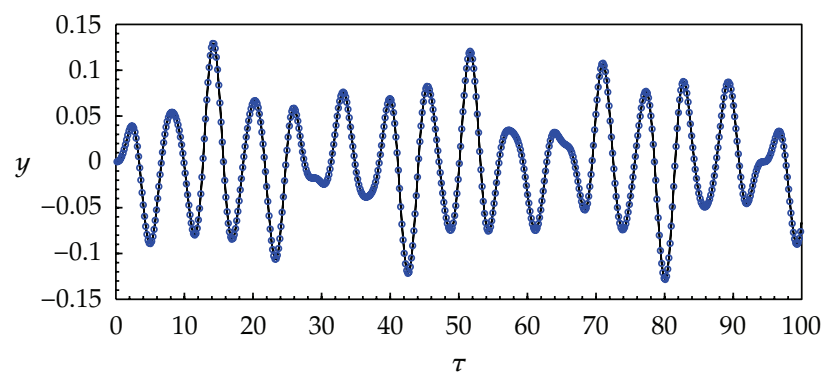

(a)

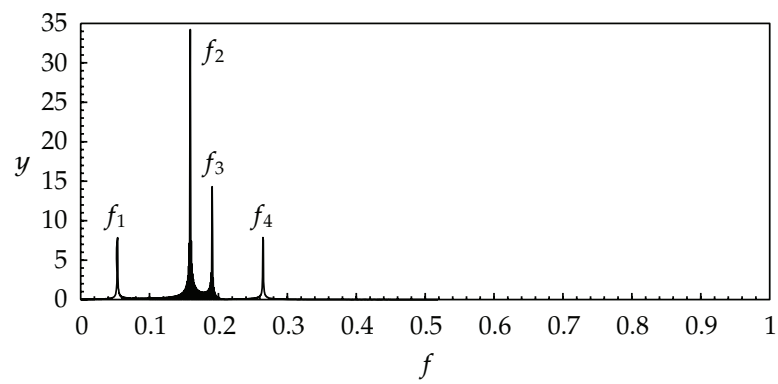

- Approximate solution

— Numerical solution

(b)

Figure 6: The nondimensional response and its frequency spectrum in $y$ direction when $E=0.03$ : (a) response; (b) spectrum.

space, and hence the three second-order equations can be converted easily to six first-order ones

$$
\begin{aligned}
& X^{\prime}=\eta, \quad Y^{\prime}=\xi, \quad \theta^{\prime}=\vartheta, \\
& \begin{aligned}
\eta^{\prime}=\frac{1}{\left(1+\sin ^{2} \theta\right)}\left[L \vartheta^{2} \sin \theta \cos \tau+2 L \vartheta \cos \theta \sin \tau-L \vartheta \cos ^{3} \theta \sin \tau\right. \\
\left.-\left(\omega_{0}^{2}-1\right) L \sin ^{3} \theta \cos \tau-\omega_{0}^{2} X\left(1+\sin ^{2} \theta\right)+\omega_{t}^{2} L \theta \cos \theta \cos \tau+E \cos \tau\right], \\
\xi^{\prime}=\frac{1}{\left(1+\sin ^{2} \theta\right)}\left[L \vartheta^{2} \sin \theta \sin \tau-2 L \vartheta \cos \theta \cos \tau+L \vartheta \cos ^{3} \theta \cos \tau\right. \\
\left.-\left(\omega_{0}^{2}-1\right) L \sin ^{3} \theta \sin \tau-\omega_{0}^{2} Y\left(1+\sin ^{2} \theta\right)+\omega_{t}^{2} L \theta \cos \theta \sin \tau+E \sin \tau\right], \\
\vartheta^{\prime}=-\frac{1}{\left(1+\sin ^{2} \theta\right)}\left[\vartheta^{2} \sin \theta \cos \theta+\left(\omega_{0}^{2}-1\right) \sin \theta \cos \theta+2 \omega_{t}^{2} \theta+\frac{E}{L} \cos \theta\right] .
\end{aligned}
\end{aligned}
$$

For the following calculation, the values of systematic parameters are considered as $\omega_{0}=1.20, \omega_{t}=0.02$, and $L=20$, and all the initial generalized displacements and velocities are set to zero.

Figures 3(a) and 4(a) depict the displacement responses of rotor system in $x$ and $y$ directions when $E=0.05$, respectively, in which the curves are demonstrated by using MMS 


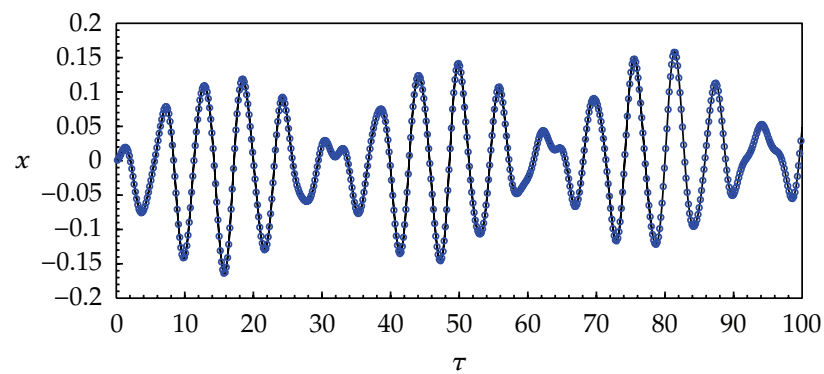

(a)

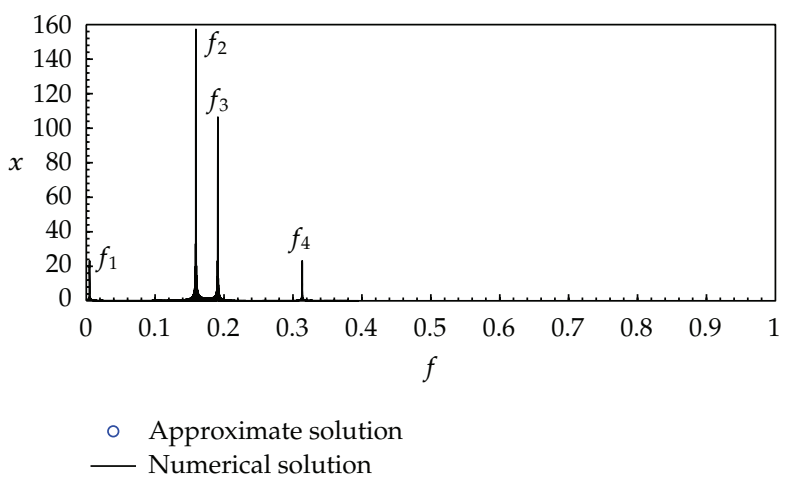

(b)

Figure 7: The nondimensional response and its frequency spectrum in $x$ direction when $\omega_{0}=1.20, \omega_{t}=$ 0.50 , and $E=0.05$ : (a) response; (b) spectrum.

and the numerical technique. The results show that the agreements between their responses are pretty almost at each interval, and thereby it proves that the dynamic behaviors of the system are in the form of (3.17) under certain initial conditions. Figures 3(b) and 4(b) appear as the frequency spectra of displacement responses by the numerical method, in which there are mainly four components from the vibrating frequencies: $f_{1}=(\omega-1) / 2 \pi=0.05349, f_{2}=$ $1 / 2 \pi=0.15915, f_{3}=\omega_{0} / 2 \pi=0.19099$, and $f_{4}=(\omega+1) / 2 \pi=0.26482$. Actually, it reveals that the synchronous motion $f_{2}$ emerges in the responses, which are obviously from the mass unbalance, and $f_{3}$ is the natural frequency of the derived linear system as shown in (3.15), $f_{1}$ and $f_{4}$ are the combinations between $\omega / 2 \pi$ and the rotating speed $\Omega$, which occur frequently for a nonlinear system. Figures 5 and 6 also show the relevant responses and the spectra when $E=0.03$. Figures 7 and 8 illustrate the responses and their spectra when $E=0.05$, and $\omega_{0}=1.20, \omega_{t}=0.50$, namely, the angular stiffness of flexible coupling increases these vibration characteristics display the multifrequency signatures in the rotor system connected a flexible coupling with a dynamic angular misalignment.

\section{Conclusions}

The misalignment of rotor system is an important reason which causes mechanical vibration; a dynamic misalignment or offset of the rotors can be produced at work even the system is aligned under the static conditions. In this paper, firstly a mathematic model of the multirotor 


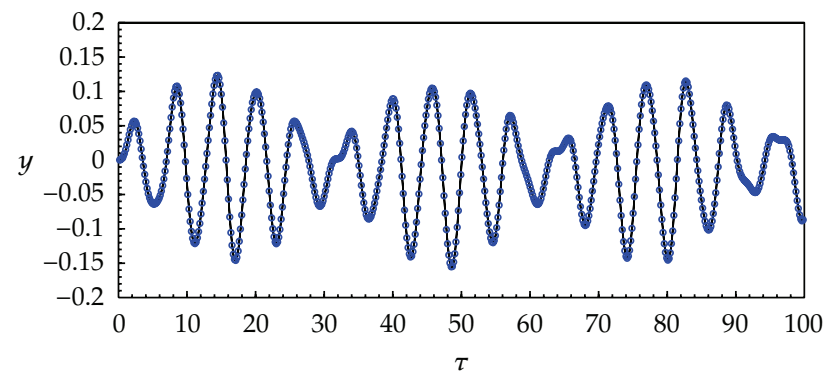

(a)

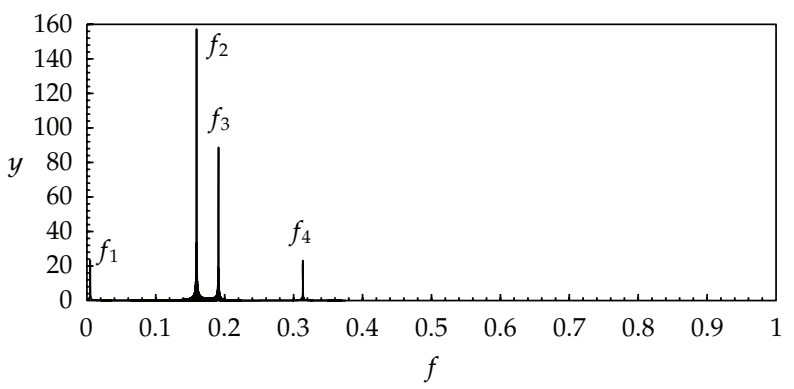

- Approximate solution

- Numerical solution

(b)

Figure 8: The nondimensional response and its frequency spectrum in $y$ direction when $\omega_{0}=1.20, \omega_{t}=$ 0.50 , and $E=0.05$ : (a) response; (b) spectrum.

system with a flexible coupling is established after considering the effect on a dynamic angular misalignment based on Lagrange's equation, and it shows that the system is of a parametric oscillation with strongly nonlinear characteristics. Then, the method of multiple scales and Runge-Kutta numerical technique are carried out, respectively. And the results indicate that the responses in lateral direction consist of some parts, that is, the synchronous vibration due to the mass unbalance, the free vibration at natural frequency of the derived linear system, and the angular motions caused by the flexible coupling at the combined frequencies.

\section{Acknowledgment}

The author acknowledges the financial support from the National Natural Science Foundation of China, China (Grant no. 11072190).

\section{References}

[1] C. B. Gibbons, "Coupling misalignment forces," in Proceedings of the 5th Turbo-Machinery Symposium, pp. 111-116, Gas Turbine Laboratories, Texas A\&M University, 1976.

[2] D. L. Dewell and L. D. Mitchell, "Detection of a misalignment disk coupling using spectrum analysis," ASME Journal of Vibration, Acoustics, Stress, and Reliability in Design, vol. 106, no. 1, pp. 9-16, 1984.

[3] M. Li and L. Yu, "Analysis of the coupled lateral torsional vibration of a rotor-bearing system with a misaligned gear coupling," Journal of Sound and Vibration, vol. 243, no. 2, pp. 283-300, 2001. 
[4] K. M. Al-Hussain and I. Redmond, "Dynamic response of two rotors connected by rigid mechanical coupling with parallel misalignment," Journal of Sound and Vibration, vol. 249, no. 3, pp. 483-498, 2002.

[5] K. M. Al-Hussain, "Dynamic stability of two rigid rotors connected by a flexible coupling with angular misalignment," Journal of Sound and Vibration, vol. 266, no. 2, pp. 217-234, 2003.

[6] S. Prabhakar, A. S. Sekhar, and A. R. Mohanty, "Vibration analysis of a misaligned rotor-couplingbearing system passing through the critical speed," Proceedings of the Institution of Mechanical Engineers Part C, vol. 215, no. 12, pp. 1417-1428, 2001.

[7] M. A. Hili, T. Fakhfakh, and M. Haddar, "Failure analysis of a misaligned and unbalanced flexible rotor," Journal of Failure Analysis and Prevention, vol. 6, no. 4, pp. 73-82, 2006.

[8] A. W. Lees, "Misalignment in rigidly coupled rotors," Journal of Sound and Vibration, vol. 305, no. 1-2, pp. 261-271, 2007.

[9] M. Li, "Nonlinear dynamics of a rotor system subject to a holonomic constraint of parallel misalignment," Dynamics of Continuous, Discrete and Impulsive Systems Series B, vol. 14, no. 5, pp. 222 226, 2007.

[10] B. Slim, A. H. Molka, M. Mohamed, F. Taher, and H. Mohamed, "Dynamic behaviour of hydrodynamic journal bearings in presence of rotor spatial angular misalignment," Mechanism and Machine Theory, vol. 44, no. 8, pp. 1548-1559, 2009.

[11] T. H. Patel and A. K. Darpe, "Vibration response of misaligned rotors," Journal of Sound and Vibration, vol. 325, no. 3, pp. 609-628, 2009.

[12] T. H. Patel and A. K. Darpe, "Experimental investigations on vibration response of misaligned rotors," Mechanical Systems and Signal Processing, vol. 23, no. 7, pp. 2236-2252, 2009.

[13] I. Redmond, "Study of a misaligned flexibly coupled shaft system having nonlinear bearings and cyclic coupling stiffness-theoretical model and analysis," Journal of Sound and Vibration, vol. 329, no. 6, pp. 700-720, 2010.

[14] G. Zhao, Y. Yu, S. Gao, T. Sun, and Z. Liu, "Study on dynamic misalignment of coupled rotor-coupling-isolator system in marine power plant," Proceedings of the Asia-Pacific Power and Energy Engineering Conference (APPEEC '11), Wuhan, China, 2011.

[15] A. H. Nayfeh and D. T. Mook, Nonlinear Oscillations, Wiley, New York, NY, USA, 1979. 


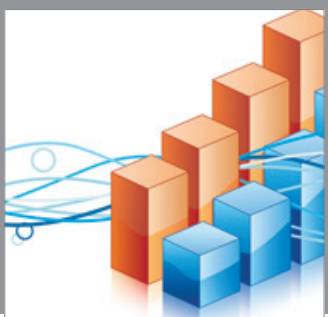

Advances in

Operations Research

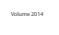

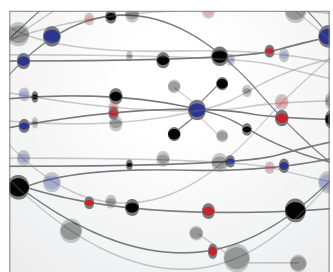

\section{The Scientific} World Journal
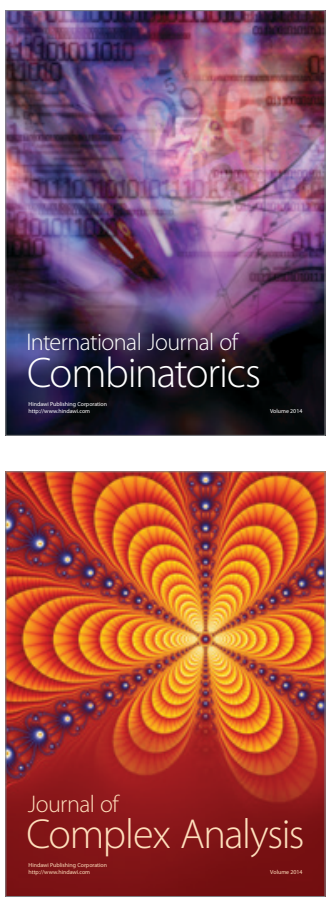

International Journal of

Mathematics and

Mathematical

Sciences
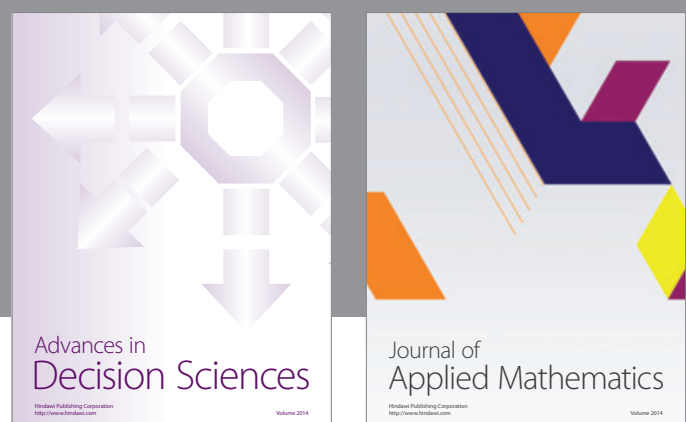

Journal of

Applied Mathematics
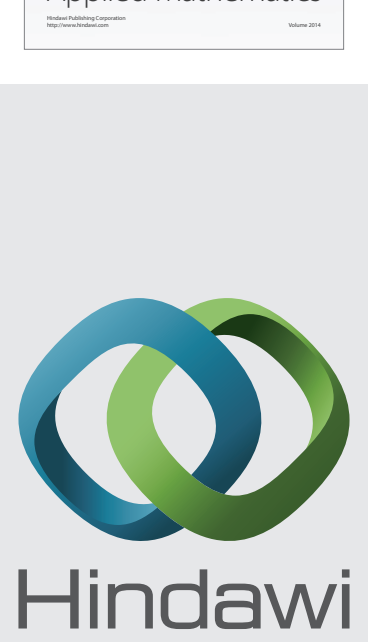

Submit your manuscripts at http://www.hindawi.com
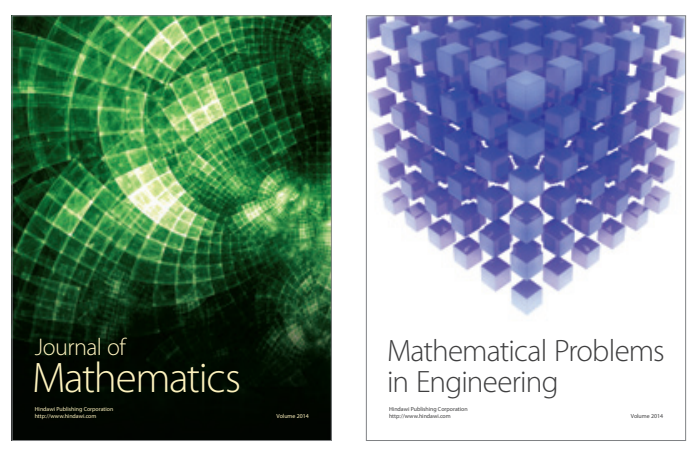

Mathematical Problems in Engineering
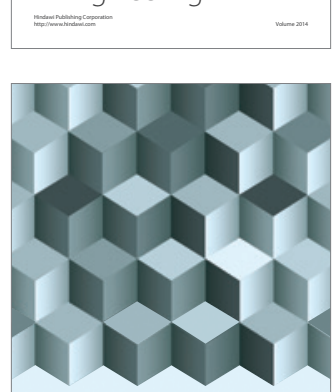

Journal of

Function Spaces
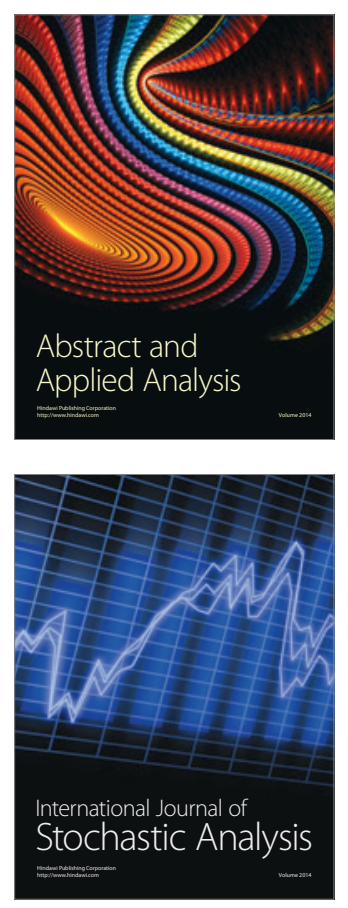

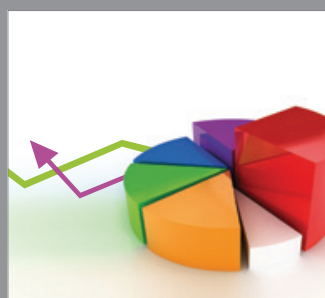

ournal of

Probability and Statistics

Promensencen
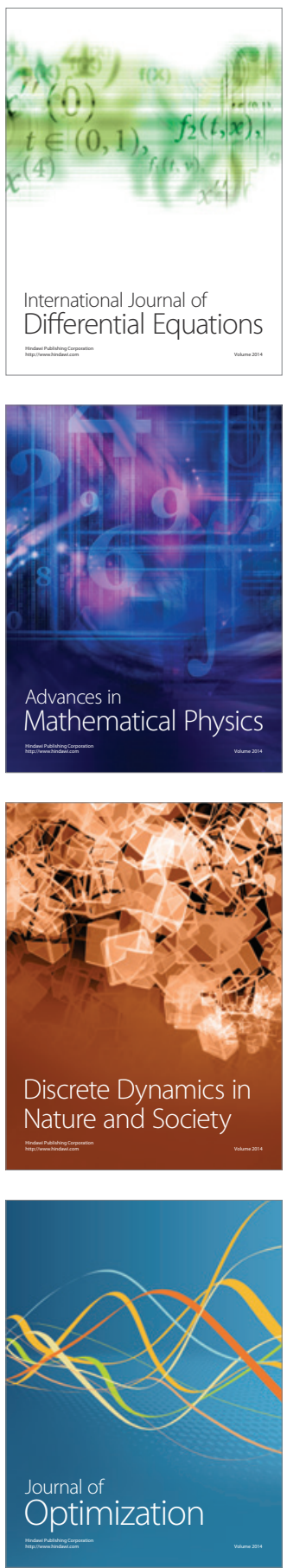